\title{
Invo-regular unital rings
}

\begin{abstract}
It was asked by Nicholson (Comm. Algebra, 1999) whether or not unit-regular rings are themselves strongly clean. Although they are clean as proved by Camillo-Khurana (Comm. Algebra, 2001), recently Nielsen and Šter showed in Trans. Amer. Math. Soc., 2018 that there exists a unit-regular ring which is not strongly clean. However, we define here a proper subclass of rings of the class of unit-regular rings, called invo-regular rings, and establish that they are strongly clean. Interestingly, without any concrete indications a priori, these rings are manifestly even commutative invo-clean as defined by the author in Commun. Korean Math. Soc., 2017.
\end{abstract}

1. Introduction and background. Everywhere in the text of the present article, all rings are assumed to be associative, containing the identity element 1 which, in general, differs from the zero element 0 . Our terminology and notations are mainly in agreement with those from [7] and [10]. For instance, for such a ring $R$, the symbol $U(R)$ stands for the group of units, $\operatorname{Inv}(R)$ for the set of all involutions (= square roots of 1$), \operatorname{Id}(R)$ for the set of all idempotents and $\operatorname{Nil}(R)$ for the set of all nilpotents.

In [6] there was introduced the following important notion.

Definition 1.1. A ring $R$ is said to be unit-regular if, for each $r \in R$, there is $u \in U(R)$ such that $r=$ rur.

2010 Mathematics Subject Classification. 16D60, 16S34, 16U60.

Key words and phrases. Unit-regular rings, clean rings, strongly clean rings, idempotents, involutions, nilpotents, units. 
Since the equality $r=$ rur is always tantamount to the condition that $u r$ is an idempotent, say $e$, it is apparent that $r=u^{-1} e$, and conversely.

The following also appeared in [6].

Definition 1.2. A ring $R$ is said to be strongly regular if, for each $r \in R$, there is $u \in U(R)$ such that $r=r^{2} u$, i.e., $r=$ rur with $u r=r u$.

It is well known that all strongly regular rings are unit-regular (see, for instance, [6] or [7]), but the converse fails in general. In fact, all elements $r$ in strongly regular rings can be written also like this $r=w f=f w$ for some $w \in U(R)$ and $f \in \operatorname{Id}(R)$.

On the other hand, in [12] was defined the following famous concept.

Definition 1.3. A ring $R$ is called clean if, for every $r \in R$, there are $u \in U(R)$ and $e \in I d(R)$ with $r=u+e$. If, in addition, the commutativity condition $u e=e u$ is satisfied, the clean $\operatorname{ring} R$ is said to be strongly-clean.

There was shown in [1] the fundamental fact that unit-regular rings are necessarily clean. Furthermore, Nicholson (cf. [13]) asked if a unit-regular ring is even strongly clean. Recently, this was answered in [14] in the negative by constructing a very special unit-regular ring which is, in fact, not strongly clean.

The aim of the current paper is to take into account the specific nature of this example from [14] and to prove that there are quite natural proper subclasses of unit-regular rings which are strongly clean. This will be done in the subsequent section. We terminate the paper with some left-open problems.

2. Main definition and result. We first begin with our key instruments.

Definition 2.1. ([3]) A ring $R$ is called strongly invo-regular if, for each $r \in R$, there exists $v \in \operatorname{Inv}(R)$ such that $r=r^{2} v$, that is, $r^{2}=r v$.

It was obtained in [3, Theorem 2.2] that a ring $R$ is strongly invo-regular if, and only if, $R \subseteq \prod_{\lambda} \mathbb{Z}_{2} \times \prod_{\mu} \mathbb{Z}_{3}$ for some ordinals $\lambda$, $\mu$.

In order to get a worthwhile generalization of these rings, it is also of some interest to consider those rings $R$ for which either $r^{2}-r v \in \operatorname{Nil}(R)$ or $r^{2}-r v \in I d(R)$. However, we will proceed in another way, namely we shall now slightly extend Definition 2.1 to the next one as follows:

Definition 2.2. A ring $R$ is said to be invo-regular if, for every $r \in R$, there exists $v \in \operatorname{Inv}(R)$ such that $r=r v r$, that is, $r=v e$, where $e=v r \in \operatorname{Id}(R)$.

It is straightforward to see that a ring $R$ is invo-regular exactly when $R$ is unit-regular and $U(R)=\operatorname{Inv}(R)$.

Recall that, imitating [4], a ring $R$ is called invo-clean if any $r \in R$ can be represented as $r=v+e$, where $v \in \operatorname{Inv}(R)$ and $e \in \operatorname{Id}(R)$.

Proposition 2.3. Invo-regular rings are invo-clean. 
Proof. Since invo-regular rings are unit-regular, we appeal to [1] to get that they are clean. However, as already commented above, all units being involutions imply that invo-regular rings have to be invo-clean, as claimed.

We now need some more technicalities.

Lemma 2.4. In any invo-regular ring $6=0$.

Proof. Letting $2 \in R$ for some invo-regular ring $R$, it must be that $2=v e$ for some $v \in \operatorname{Inv}(R)$ and $e \in \operatorname{Id}(R)$. Thus $2 v=e$ and squaring we have $4=e=2 v$. Again squaring, we obtain that $16=4$, i.e., $12=0$. Hence $6^{2}=3.12=0$ and so $6 \in \operatorname{Nil}(R)$. Since the Jacobson radical of unit-regular rings is always zero (see, e.g., [7]), this enables us that $6 \in J(R)=\{0\}$, as expected.

Proposition 2.5. A ring $R$ is invo-regular if, and only if, $R \cong R_{1} \times R_{2}$, where $R_{1}$ is an invo-regular ring of characteristic 2 and $R_{2}$ is an invo-regular ring of characteristic 3 .

Proof. Since $(2,3)=1$ and by Lemma 2.4 we have $6=0$, a standard trick works to deduce that $R=2 R \oplus 3 R$. Hence $R / 2 R \cong 3 R$ and $R / 3 R \cong 2 R$ which both imply that $R \cong R_{1} \times R_{2}$, where $R_{1}=R / 2 R$ and $R_{2}=R / 3 R$. But it is not too hard to check that homomorphic images of an invo-regular ring are again invo-regular rings, which gives our claim.

We now arrive at the following central result as, surprisingly, the following holds.

Theorem 2.6. The following three items are equivalent:

(i) $R$ is invo-regular.

(ii) $R$ is strongly invo-regular.

(iii) $R$ is a subdirect product of copies of the fields $\mathbb{Z}_{2}$ and $\mathbb{Z}_{3}$.

Proof. The implication (ii) $\Rightarrow$ (i) is obvious. The equivalence (ii) $\Longleftrightarrow$ (iii) was proved in [3]. That is why we will deal now only with the implication (i) $\Rightarrow$ (iii). To that goal, with Proposition 2.5 at hand, one can write $R \cong R_{1} \times R_{2}$, where both $R_{1}, R_{2}$ are invo-regular and $\operatorname{char}\left(R_{1}\right)=2$ whereas $\operatorname{char}\left(R_{2}\right)=3$.

In the first case, because of the equality $U\left(R_{1}\right)=\operatorname{Inv}\left(R_{1}\right)$, it follows that $U\left(R_{1}\right)=1+\operatorname{Nil}\left(R_{1}\right)$ as $\left(1+\operatorname{Inv}\left(R_{1}\right)\right)^{2}=1+\operatorname{Inv}^{2}\left(R_{1}\right)=0$. Thus $R_{1}$ is a UU ring (i.e. all units are unipotent units). However, it was proved in [2], and independently in [5], that unit-regular UU rings are Boolean. Consequently, $R_{1}$ must be Boolean, as asserted.

In the second case, Proposition 2.3 tells us that $R_{2}$ is an invo-clean ring of characteristic 3 , whence [4, Theorem 2.15] allows us to conclude that $R_{2}$ can be embedded in a direct product of copies of the field $\mathbb{Z}_{3}$. 
Remark 2.7. Paralleling a similar argumentation to that from [5], one can say something more as follows: If point (iii) is true, then $R$ is a commutative regular ring and every unit of $R$ is an involution, so that the implications "(iii) $\Rightarrow$ (ii) $\Rightarrow$ (i)" follow immediately. As for the remaining one "(i) $\Rightarrow$ (iii)", we process thus: For any $a \in R$, write $a=e v, e^{2}=e$ and $v^{2}=1$. Then $a^{3}=a$ in case $e$ is central. So, it suffices to prove that $R$ is reduced whence it will be necessarily abelian. In fact, if $R$ has a non-zero, squarezero element $a$, then by a well-known classical result in ring theory, mainly attributed to Levitzki (see, e.g., [5]), $R$ will contain a corner subring $e R e$ isomorphic to a full 2 by 2 matrix ring over a non-trivial ring. Thus, owing to the specific nature of units in matrix rings, it is plainly checked that $e R e$ contains a unit $u$ whose square is not $e$ and, therefore, $u+1-e$ is a unit of $R$ that is not an involution observing that $(u+1-e)^{2}=u^{2}+1-e$ (compare with Proposition 2.16, too). This is, however, the wanted contradiction.

We continue with some uniqueness in the class of invo-regular rings, defined like this:

Definition 2.8. A ring $R$ is said to be uniquely invo-regular if, for every $0 \neq r \in R$, there is a unique $v \in \operatorname{Inv}(R)$ such that $r=r v r$.

Since as we have seen above $r$ can be written as $r=v e$, where $e=v r \in$ $I d(R)$, a question which immediately arises is whether or not the uniqueness of $v$ is retained in this record as well. Specifically, the following simple but useful assertion is true.

Proposition 2.9. The ring $R$ is uniquely invo-regular if, and only if, for each $0 \neq r \in R$, there is a unique $v \in \operatorname{Inv}(R)$ with $r=$ ve for some $e \in \operatorname{Id}(R)$.

Proof. " $\Rightarrow$ ". Writing $r=v e=w f$ for some $v, w \in \operatorname{Inv}(R)$ and $e, f \in$ $I d(R)$, it follows that $v r=e$ and $w r=f$. So, vrvr $=v r$ and $w r w r=w r$, that is, $r v r=r=r w r$. This yields that $v=w$ (whence $e=f$ ), as required.

" $\Leftarrow$ ". Letting $r=r v r=r w r$ for some $v, w \in \operatorname{Inv}(R)$, it follows that $r=v e=w f$ for $e=v r \in I d(R)$ and $f=w r \in I d(R)$. This implies that $v=w$ (and hence $e=f$ ), as needed.

We are now ready to establish the following more comprehensive result, by using a technique developed in [8].

Theorem 2.10. $A$ ring $R$ is uniquely invo-regular if, and only if, either $R \cong B$, where $B$ is a Boolean ring, or $R \cong \mathbb{Z}_{3}$.

Proof. The sufficiency being trivial, we concentrate on the necessity. We claim that if $R$ is uniquely invo-regular, then $R$ is either a Boolean ring or a division ring. First of all, we will detect that all elements of $R$ are either idempotents or involutions. And so, for an arbitrary $a \in R$ we write $a v a=a$ for some unique $v$ with $v^{2}=1$, and assume that $a^{2} \neq a$ (i.e., $v \neq 1$ ). We 
will show that $a^{2}=1$. In fact, notice that all units in $R$ are involutions as well as we can represent the element $a$ as follows:

$$
a[v(1-a(1-a v))] a=a v a=a[(1-(1-v a) a) v] a,
$$

where $(1-a(1-a v))^{-1}=1+a(1-a v)$ and $(1-(1-v a) a)^{-1}=1+(1-v a) a$ because $a(1-v a) a=a(1-a v) a=0$. The uniqueness now yields that

$$
v(1-a(1-a v))=v=(1-(1-v a) a) v,
$$

that is,

$$
a(1-a v)=0=(1-v a) a .
$$

The last equalities ensure that $a^{2} v=a=v a^{2}$, i.e., $a v=a^{2}=v a$. This means that $a^{3}=a$ and hence $a^{4}=a^{2}$. Therefore, one may verify that $\left(a+1-a^{2}\right)^{2}=1$ and $\left(1-a^{2}\right)^{2}=1-a^{2}$. But the relationships

$$
a\left(a+1-a^{2}\right) a=a=a v a
$$

lead us to $v=a+1-a^{2}$ whence $v\left(1-a^{2}\right)=\left(a+1-a^{2}\right)\left(1-a^{2}\right)=1-a^{2}$. If now $a^{2} \neq 1$, by what we have just shown above, we infer that

$$
\left(1-a^{2}\right) v\left(1-a^{2}\right)=1-a^{2}=\left(1-a^{2}\right) \cdot 1 \cdot\left(1-a^{2}\right) .
$$

The uniqueness assures that $v=1$, a contradiction. This substantiates that $a^{2}=1$, as promised.

Next, to argue the initial claim, suppose $R$ is not Boolean. So, there is $b \in R$ with $b^{2} \neq b$. By what we have already established, $b^{2}=1$. Let $x \in R \backslash\{0\}$ and consider $b x$.

Case 1: $(b x)^{2}=b x$. Thus $b x b x=b x$ gives $x b x=x$. But $x^{2} \neq x$ as otherwise $x=x .1 . x$ and so the uniqueness forces $b=1$ and hence $b^{2}=$ $b$, contrary to our assumption. Finally, in view of our conclusions above, $x^{2}=1$.

Case 2: $(b x)^{2} \neq b x$. So $(b x)^{2}=1$, which means that $b x b x=1$, that is, $x b x=b$. Therefore, since $b$ is invertible with the inverse $b$ being an involution, it readily follows that $x$ is invertible with the inverse $b x b$. This finalizes our claim at the beginning of the proof that $R$ is a division ring.

Furthermore, utilizing Theorem 2.6 alluded to above, let us assume that $R$ is not Boolean. Thus, it follows from the first part above that $R$ is a division ring and, resultantly, being indecomposable, $R$ is a subdirect product of the $\mathbb{Z}_{3}$ 's. It is easily seen that each element $y \in R$ now satisfies the equation $y^{3}=y$. If $y \neq 0$, then $y$ inverts and so $y^{2}=1$, which amounts to $(y-1)(y+1)=0$. If $y \neq 1$, we have $y=-1$ as well as if $y \neq-1$, we have $y=1$. This guarantees that $R \cong \mathbb{Z}_{3}$, as stated.

Remark 2.11. Certainly, utilizing Theorem 2.6, the proof of the previous theorem can be simplified like this: It is quickly observed that $(1-a v) a v=0$ since $a v a=a$, and hence we deduce $(1-a v) a=0$, implying that $(1-v a) a=$ 0 because $R$ is a commutative ring being invo-regular. 
Moreover, a sketch of a parallel proof of the preceding theorem, by the usage of another arguments, could be the following one: One only needs to show the necessity. By part (iii) of Theorem 2.6, it follows that $x^{3}=x$ for every $x \in R$. So $x^{2}$ is an idempotent for each $x \in R$. Since the case $2=0$ obviously leads us to the obtaining of a Boolean ring, one can assume that $\operatorname{char}(R)=3$ or, in other words, that $R$ is a subdirect product of isomorphic copies of the field $\mathbb{Z}_{3}$. Thus, to prove that $R \cong \mathbb{Z}_{3}$, it suffices to establish that $R$ has no non-trivial idempotents. In doing that, let $e \neq 0,1$ be an arbitrary idempotent. Then one verifies that the equalities $-e=e(1-2 e) e=e(-1) e$ hold, where $1-2 e=1+e$ and -1 are distinct involutions as $3=0$. This is a contradiction, however.

Mimicking the same idea for proof as that from the preceding theorem, we can find a necessary and sufficient condition when for the strongly regular ring $R$ the next additional condition is valid: For any $r \in R$ there is a unique $v \in \operatorname{Inv}(R)$ such that $r=r^{2} v$. The expected result, which can be proved in a way of similarity to that quoted above, will be again either the Boolean ring or the three element field.

Another way to consider uniqueness is the following one:

Definition 2.12. A ring $R$ is called pseudo uniquely invo-regular if, for each $r \in R$, there is a unique $e \in \operatorname{Id}(R)$ with $r=v e$ for some $v \in \operatorname{Inv}(R)$.

So, we have now at our disposal all the ingredients necessary to prove the following rather surprising statement.

Theorem 2.13. A ring $R$ is pseudo uniquely invo-regular if, and only if, it is invo-regular.

Proof. One way being trivial, we concentrate on the other one. To this aim, given $R$ is an invo-regular ring. Appealing to Theorem 2.6, accomplishing it with [9], we deduce that $R$ is a commutative ring and all elements $y$ in $R$ satisfy the equation $y^{3}=y$ which amounts to $y=v e$, where $v=$ $-y^{2}+y+1 \in \operatorname{Inv}(R)$ and $e=y^{2} \in \operatorname{Id}(R)$. Supposing $y=v e=w f$, for some $w \in \operatorname{Inv}(R)$ and $f \in \operatorname{Id}(R)$, with the commutativity in mind we detect that $y^{2}=v^{2} e^{2}=w^{2} f^{2}$, i.e., $y^{2}=e=f$, as expected.

The following note is helpful.

Remark 2.14. Notice that in the above decomposition $y=v e=w f$, although we concluded $e=f$, it could be that $v \neq w$ as the next example unambiguously illustrates: Let $R=\mathbb{Z}_{3} \times \mathbb{Z}_{3}$ and consider the element $(-1,0)$. It can be twicer written as $(-1,0)=(-1,1)(1,0)=(-1,-1)(1,0)$, so that by substituting $v=(-1,1) \neq(-1,-1)=w$ and $e=(1,0)=f$ the claim sustained.

We continue with certain element-wise properties of (strongly) invo-regular elements. Imitating Definition 2.1 (see cf. [3]), an element $a$ from a ring $R$ is 
called strongly invo-regular if there is $v \in \operatorname{Inv}(R)$ such that $a=a^{2} v=v a^{2}$. A natural question, regarding this element $a$, is whether or not it can be presented as a strongly invo-clean element (e.g., [4]), which is of the form $a=w+f$, where $w \in \operatorname{Inv}(R)$ and $f \in \operatorname{Id}(R)$ with $w f=f w$. The answer is the positive "yes" and is subsumed by the following.

Proposition 2.15. Any strongly invo-regular element is strongly invo-clean.

Proof. It follows directly from the definition that $a=a^{2} v=v a^{2}$ and $v a=a v=a^{2}$ for some $v \in \operatorname{Inv}(R)$. Writing $a=e v=v e$, where $e=a^{2}=e^{2}$, one observes that $a=(v e+e-1)+(1-e)$. What remains to show is that $w=v e+e-1$ is an involution, because $f=1-e$ is always an idempotent which commutes with $w$. Indeed, squaring $w^{2}=(v e)^{2}+e+1+2 v e^{2}-2 v e-2 e$ and taking into account that $(v e)^{2}=v^{2} e^{2}=e$ and $e^{2}=e$, we obtain the wanted equality that $w^{2}=1$.

We will now examine how invo-regularity is situated in the corner rings. It is well known that (see, e.g., [11]) that if $R$ is a unit-regular ring, then the corner subring $e R e$ is also unit-regular for any $e \in I d(R)$. We will now prove the following similarity.

Proposition 2.16. If $R$ is an invo-regular ring and $e \in \operatorname{Id}(R)$, then eRe is an invo-regular ring, too.

Proof. By the comments above, $e$ Re is unit-regular. But we shall show that $U(e R e)=\operatorname{Inv}(e R e)$ which is enough to conclude that $e R e$ is invo-regular. To that purpose, letting $u \in U(e R e)$, we obtain that $u+1-e \in U(R)$ with the inverse $u^{\prime}+1-e$, where $u u^{\prime}=u^{\prime} u=e$. However, $u(1-e)=(1-e) u=0$ and so $1=(u+1-e)^{2}=u^{2}+(1-e)^{2}=u^{2}+1-e$. This insures that $u^{2}=e$, as required.

We finish off our work with three queries of interest and importance.

Problem 2.17. If $R$ is a ring and $e \in I d(R)$ such that $e R e \cong(1-e) R(1-$ $e) \cong \mathbb{Z}_{2}$, is it true that $R$ is unit-regular?

For some arbitrary but a fixed $n \in \mathbb{N}$, we will say that a ring $R$ is $n$-torsion regular if, for any $r$, there exists $w \in U(R)$ with $w^{n}=1$, such that $r=r w r$. It is self-evident that $n$-torsion regular rings are of necessity unit-regular with bounded by $n$ unit groups.

Problem 2.18. Does it follow that $n$-torsion regular rings are strongly clean?

It is worthwhile noticing that if $R$ is a $p$-torsion regular ring of prime $\operatorname{char}(R)=p$, then $R$ has to be Boolean. Indeed, for every $u \in U(R)$ we have $u^{p}=1$ and so $u=1+(u-1) \in 1+\operatorname{Nil}(R)$ bearing in mind that $(u-1)^{p}=u^{p}-1=0$. This allows us to derive that $R$ is a unit-regular UU 
ring and, as we already have seen above, the application of [2] or [5] enables us that $R$ is necessarily Boolean, as pursued.

And so, we close the queries with the following element-wise question.

Problem 2.19. Is an invo-regular element in an arbitrary ring a clean element in this ring?

In fact, it can be written for such an element $r$ in a ring $R$ that $r=e v=$ $v f$ for some $e, f \in \operatorname{Id}(R)$ and $v \in \operatorname{Inv}(R)$ with $v(e+f)=(e+f) v$. It is worth noticing that this question has a negative resolution for unit-regular elements (cf. [14]). So, for a possible counterexample to this query, one could look at the ring $R=\mathbb{M}_{2}(\mathbb{Z})$ which is manifestly not clean as the ring of integers $\mathbb{Z}$ is not clean.

Now, for an arbitrary but fixed $n \in \mathbb{N}$, we will say that a $\operatorname{ring} R$ is strongly $n$-torsion regular if, for any $r$, there exists $w \in U(R)$ with $w^{n}=1$, such that $r=r^{2} w$. It is elementarily seen that $r=r^{n+1}$ and hence, owing to the famous theorem of Jacobson, strongly $n$-torsion regular rings are of necessity commutative. So, we come to

Problem 2.20. Characterize up to an isomorphism strongly $n$-torsion regular rings.

As a final challenging problem, we state:

Problem 2.21. Characterize those rings $R$ for which, for each $r \in R$, there exists $e \in \operatorname{Id}(R) \cap[\operatorname{Inv}(R) r]$ such that $r-r e=r(1-e) \in \operatorname{Nil}(R)$.

3. Acknowledgements. The author acknowledges the referee's role in making up the paper more nearly self-contained and friendly to the reader. Sincere thanks are also due to the editors, for their professional editorial management.

\section{REFERENCES}

[1] Camillo, V. P., Khurana, D., A characterization of unit regular rings, Commun. Algebra 29 (2001), 2293-2295.

[2] Danchev, P. V., A new characterization of Boolean rings with identity, Irish Math. Soc. Bull. 76 (2015), 55-60.

[3] Danchev, P. V., On weakly clean and weakly exchange rings having the strong property, Publ. Inst. Math. Beograd 101 (2017), 135-142.

[4] Danchev, P. V., Invo-clean unital rings, Commun. Korean Math. Soc. 32 (2017), 19-27.

[5] Danchev, P. V., Lam, T. Y., Rings with unipotent units, Publ. Math. Debrecen 88 (2016), 449-466.

[6] Ehrlich, G., Unit-regular rings, Portugal. Math. 27 (1968), 209-212.

[7] Goodearl, K. R., Von Neumann Regular Rings, Second Edition, Robert E. Krieger Publishing Co., Inc., Malabar, FL, 1991.

[8] Hartwig, R. E., Luh, J., A note on the group structure of unit regular ring elements, Pacific J. Math. 71 (1977), 449-461. 
[9] Hirano, Y., Tominaga, H., Rings in which every element is the sum of two idempotents, Bull. Austral. Math. Soc. 37 (1988), 161-164.

[10] Lam, T. Y., A First Course in Noncommutative Rings, Second Edition, SpringerVerlag, Berlin-Heidelberg-New York, 2001.

[11] Lam, T. Y., Murray, W., Unit regular elements in corner rings, Bull. Hong Kong Math. Soc. 1 (1997), 61-65.

[12] Nicholson, W. K., Lifting idempotents and exchange rings, Trans. Amer. Math. Soc. 229 (1977), 269-278.

[13] Nicholson, W. K., Strongly clean rings and Fitting's lemma, Commun. Algebra 27 (1999), 3583-3592.

[14] Nielsen, P. P., Ster, J., Connections between unit-regularity, regularity, cleanness and strong cleanness of elements and rings, Trans. Amer. Math. Soc. 370 (2018), 1759-1782.

Peter V. Danchev

Institute of Mathematics \& Informatics

Bulgarian Academy of Sciences

"Acad. G. Bonchev" str., bl. 8

1113 Sofia

Bulgaria

e-mail: danchev@math.bas.bg; pvdanchev@yahoo.com

Received September 11, 2017 